\title{
George Mackey and His Work on Representation Theory and Foundations of Physics
}

\author{
V. S. Varadarajan \\ To the memory of George Mackey
}

\begin{abstract}
This article is a retrospective view of the work of George Mackey and its impact on the mathematics of his time and ours. The principal themes of his work-systems of imprimitivity, induced representations, projective representations, Borel cohomology, metaplectic representations, and so on, are examined and shown to have been central in the development of the theory of unitary representations of arbitrary separable locally compact groups. Also examined are his contributions to the foundations of quantum mechanics, in particular the circle of ideas that led to the famous Mackey-Gleason theorem and a significant sharpening of von Neumann's theorem on the impossibility of obtaining the results of quantum mechanics by a mechanism of hidden variables.
\end{abstract}

\section{Contents}

1. Introduction

2. Stone-Von Neumann theorem, systems of imprimitivity, and the imprimitivity theorem

3. Semi direct products and the little group method. Representations of finite length and orbit schemes

4. Super Lie groups and their systems of imprimitivity

5. Intertwining numbers, double coset decompositions, and irreducibility of induced representations

6. Projective representations, Borel cohomology of groups, and the metaplectic representation

7. Foundations of Physics: hidden variables and the Mackey-Gleason theorem

2000 Mathematics Subject Classification. 22D10, 81P10.

Key words and phrases. Stone-von Neumann theorem, systems of imprimitivity, unitary representations, induced representations, semi direct products, super Lie groups, intertwining numbers, double coset decompositions, projective representations, Borel cohomology, Baruer groups, metaplectic representation, hidden variables, Mackey-Gleason theorem, quantum logics, canonical commutation rules, quantum information theory, quantum stochastic calculus.

(C)0000 (copyright holder) 


\section{Introduction}

It is my aim here to speak about George W. Mackey, discuss some of his ideas and their impact on the mathematics of his time, and its continuing influence on the themes and concerns of our own era, a generation and more removed from his. His influence went beyond the immediate circle of his students and their students, and percolated to a very wide circle of mathematicians. The themes he initiated played a very important role in shaping the agenda of a large part of contemporary mathematics - more precisely, functional analysis, representation theory of general locally compact groups, ergodic theory, and mathematical physics, all under the umbrella of representation theory of general locally compact groups and their homogeneous spaces. He was a giant in a milieu that included men like Gel'fand, Godement, Harish-Chandra, Mostow, Selberg, Langlands, Kostant, and a host of others, a milieu in which he more than held his own and made beautiful and permanent discoveries that rivaled those of his contemporaries, and, if I may say, of their successors.

In the beginning of his career in the early and mid 1940's he worked in functional analysis, especially the theory of locally convex topological vector spaces which was just emerging at that time. He was the co-discoverer, along with my late friend and colleague, Richard Arens, of the so-called Mackey-Arens topology. But soon he gravitated towards unitary representation theory and the mathematics of quantum theory. In his own mind he probably saw them not as two distinct disciplines but as two facets of a complete picture. Starting from the late 1940's and reaching into the mid 1960's, he made fundamental contributions to the theory of unitary representations of locally compact groups, going beyond the classical theory where the groups were either compact or abelian. When everyone else was trying to work out the theory on Lie groups and going for intensive and detailed understanding, he opted for the broad picture and tried to see what can be done for the category of all locally compact second countable groups and their homogeneous spaces. He discovered the surprising and rather profound fact that the locally compact category could be better understood as a subcategory of the Borel category (of standard Borel groups and their homogeneous standard Borel spaces), and pioneered the measure and operator theoretic approach to geometry and analysis on homogeneous spaces that later found fuller expression in the works of Margulis, Zimmer, Ratner, Popa, and a host of others. His ideas in ergodic theory and the ergodic aspects of group actions were the first sightings of a huge new continent that Connes explored later - the theory of non commutative differential geometry. He summarized his points of view and achievements in his American Mathematical Society Colloquium lectures given in Stillwater, Oklahoma in 1961 [Ma1], although earlier versions had been circulating in the form of his famous "Chicago Lectures" [Ma2] subsequently published by the University of Chicago Press. In addition he published two small volumes in Benjamin on Induced representations and on Foundations of Quantum Mechanics [Ma3], as well as a widely circulated set of notes on classical mechanics, quantum mechanics, and representation theory that formed the basis of the education of a huge number of mathematicians including myself. I still remember my excitement when, as a visiting assistant professor in the University of Washington in Seattle, I came across these notes, and how much of an impact the global and universal view of these subjects presented in these notes had on my thinking. In his later years he wrote several expositions of his point of 
view, pedagogical as well as historical, which are very valuable for the beginner and expert alike [Ma4].

His students and their students carried on the development of his ideas discovering new things in the process. I was not a student of his in the technical sense but had a deep interaction with him at a very early point of my career that started me on what I should do. He was aware of this and told me once that he counted me as a "half student". The notion of a half student was never clarified, but I always took it to mean that I had the freedom to work in whatever area I wanted! It was in the summer of 1961 that I first met him. I was a visiting assistant professor at the University of Washington in Seattle, and he came there to give the Walker Ames lectures. He lectured every day of the week for four weeks, with ten lectures on representation theory and the remaining ten on quantum theory. The lectures were a trial run for his Colloquium lectures at Stillwater that were to be given later that summer. It was a great opportunity for me to see a great master at work, and that encounter shaped my entire mathematical career. I became a great admirer of his way of thinking that encompassed a broad picture of mathematics and physics, and emphasized concepts above brute calculation and ideas above technique. I was deeply impressed by the curiosity as well as humility with which he viewed the role of mathematics and the mathematician in the understanding, description, and interpretation of the world of phenomena around us. I had a chance to discuss things with him every day and he gave me lots of advice. I remember one such with great vividness. We were talking about the work of Harish-Chandra and he told me that it was based on a "terrifying technique of algebra, especially Lie algebras", and that if I were ambitious it should form my starting point. No one has given me better advice since then. It was also under the influence of his lectures that I wrote my paper on the logic of quantum mechanics [Va3] and subsequently the book [Va2] on the geometry of quantum theory. Years later, when I returned again to quantum foundations, my work was still animated by the themes he had introduced [CTV] [CCTV1].

In all my encounters with him there was never any intrusive manifestation of the gap in age, experience, and achievements between us. About him there was a certain transparency that was almost child-like, in the presence of which all barriers melted away. With him, in a very real and rare sense, what you saw was what you got.

In the following sections I shall give a brief and impressionistic tour of some parts of his world of ideas with which I have some familiarity, and hope to convince the reader of their power and vitality even in the new frontiers of mathematics and physics today.

\section{Stone-Von Neumann theorem, systems of imprimitivity, and the imprimitivity theorem}

The first major contribution of Mackey to representation theory was to the problem of the uniqueness of the Schrödinger representation of the Heisenberg commutation rules in quantum mechanics. Actually the Heisenberg rules are infinitesimal; the global commutation rules which represent the integrated version of these rules were obtained by Weyl (almost at the same time as Born, Heisenberg, and Jordan obtained their results; see the very interesting account of the concerned 
events in van der Waerden's historical account $[\mathbf{v W}]$, pp. 52-54). In the simplest case for the kinematics of a single quantum particle moving on a line, whose position and momentum operators are $q$ and $p$, the Heisenberg rule is

$$
[p, q]=-i \quad(\text { we assume that } \hbar=1)
$$

while the Weyl rule is

$$
e^{i a p} e^{i b q}=e^{i a b} e^{i b q} e^{i a p} .
$$

If we take the Hilbert space of states of the particle to be $L^{2}(d q)$ and $q, p$ as the (self adjoint)operators

$$
q: \psi(q) \longmapsto q \psi(q), \quad p: \psi(q) \longmapsto-i \frac{d \psi}{d q},
$$

then both the Heisenberg and Weyl rules are satisfied, with

$$
e^{i a p}: \psi(q) \longmapsto \psi(q+a), \quad e^{i b q}: \psi(q) \longmapsto e^{i b q} \psi(q) .
$$

Weyl $[\mathbf{W}]$ (see Chapter III, §16) realized that the crucial question now is whether this is the only choice possible. Since we can replace everything by a system which is the direct sum of a number of copies of this one, the uniqueness has to be asserted under the assumption that the system is irreducible(in an obvious sense). Weyl had the very fruitful idea that the correct set up was that involving two abelian groups in duality, say $A, B$ with

$$
\chi:(a, b) \longmapsto \chi(a, b)
$$

as the duality map into the unit circle, and a pair of unitary representations $U, V$ of $A, B$ respectively, satisfying

$$
U(a) V(b)=\chi(a, b) V(b) U(a) \quad((a, b) \in A \times B) .
$$

The simplest case of interest to physics is when

$$
A=B=\mathbf{R}, \quad \chi(a, b)=e^{i a b} .
$$

He could not do this case but settled for proving the uniqueness in the case

$$
A=B=\mathbf{Z}_{N}:=\mathbf{Z} / N \mathbf{Z}, \quad \chi(a, b)=e^{2 \pi i a b / N} .
$$

Stone $[\mathbf{S t}]$ and Von Neumann $[\mathbf{v N 1}$ ) proved the uniqueness directly when $A=B=$ $\mathbf{R}^{N}$ with $\chi(a, b)=e^{i\left(a_{1} b_{1}+\cdots+a_{n} b_{n}\right)}$. Mackey's 1949 paper [Ma5] showed that the theorem (properly formulated) is true when $A=G$ is an arbitrary locally compact abelian second countable group, with $B=\widehat{G}$, the dual group of $G$, and $\chi$ the natural duality between a group and its dual group. The unitary representations $U, V$ respectively of $G, \widehat{G}$ act on a Hilbert space $\mathcal{H}$ and satisfy

$$
U(a) V(\xi)=\langle a, \xi\rangle V(\xi) U(a) \quad((a, \xi) \in G \times \widehat{G}) .
$$

Mackey's theorem was that every such pair $(U, V)$ is equivalent to a direct sum of copies of the unique irreducible system where

$$
\mathcal{H}=L^{2}(G, d q), \quad U(a): \psi(q) \longmapsto \psi(q+a), \quad V(\xi): \psi(q) \longmapsto\langle q, \xi\rangle \psi(q) .
$$

Here $d q$ is a Haar measure on $G$. Mackey's work thus placed the themes of Weyl and Stone-von Neumann in their natural generality. It is remarkable that he was able to do something highly non trivial in a theme already examined in some depth by Weyl, Stone, and von Neumann. 
Although the problem is completely symmetric between $G$ and $\widehat{G}$, in practice one breaks this symmetry and starts by representing $V$ (say) in diagonal form. This means that we write

$$
V(\xi)=\int_{G}\langle q, \xi\rangle d P(q) \quad(\xi \in \widehat{G})
$$

where $P$ is the uniquely determined spectral measure of $V$. The relation between $U$ and $V$ then becomes the relation

$$
U(a) P_{E} U(a)^{-1}=P_{E-a} \quad(* *)
$$

between $U$ and $P$. Note that both $U$ and $P$ are now defined on $G$, thus eliminating $\widehat{G}$ from the picture entirely, and hence also the assumption of commutativity on $G$. Now $P$ can be explicitly written down using the Hahn-Hellinger-Wecken-Nakano theory of spectral multiplicity [Hal]. For a $\sigma$-finite measure $\mu$ on $G$ let us write $P^{\mu}$ for the projection valued measure in $L^{2}(\mu)$ given by $P_{E}^{\mu}: f(q) \longmapsto \chi_{E}(q) f(q)$ where $\chi_{E}$ is the characteristic function of the Borel set $E$. Then the spectral multiplicity theory asserts that

$$
P \simeq n_{1} P^{\mu_{1}} \oplus n_{2} P^{\mu_{2}} \oplus \ldots
$$

where the measures $\mu_{1}, \mu_{2}, \ldots$ are mutually orthogonal to each other and $n_{1}, n_{2}, \ldots$ are distinct integers, $1 \leq n_{j} \leq \infty$ (note that $\infty$ is a possible value). The measures $\mu_{j}$ are unique up to mutual absolute continuity. The relation $(* *)$ then implies that under translations by elements of $G$, for each $j$, the measure $\mu_{j}$ is changed into another mutually absolutely continuous with respect to it (or as we say, in the same measure class). But, and this is the first key observation of Mackey, there is just one measure class on $G$ invariant with respect to all translations, namely that of the Haar measure $d q$, so that we can write

$$
P \simeq n P^{d q} \quad(1 \leq n \leq \infty) .
$$

If $\mathcal{H}_{n}$ is a Hilbert space of dimension $n$, we can take $n P^{d q}$ as the projection valued measure $1 \otimes P^{d q}$ on $\mathcal{H}_{n} \otimes L^{2}(d q)$. We still have to determine all $U$ satisfying (**) with $P$ as above. Certainly

$$
U^{0}(a): f(q) \longmapsto f(q+a) \quad\left(f \in \mathcal{H}_{n} \otimes L^{2}(d q)\right)
$$

is a possibility and we write

$$
U(a)=C(a) U^{0}(a) .
$$

The representation property for $U$ now gives the relations

$$
C\left(a_{1}+a_{2}\right)=C\left(a_{1}\right)\left[U^{0}\left(a_{1}\right) C\left(a_{2}\right) U^{0}\left(a_{1}\right)^{-1}\right]
$$

showing that $C$ becomes a cocycle with values in the unitary group of $\mathcal{H}_{n} \otimes L^{2}(d q)$ with all $C(a)$ commuting with $P$. Ignoring measure theoretic subtleties we may therefore write

$$
C(a): g \otimes f(q) \longmapsto C(a, q) g \otimes f(q) \quad\left(g \in \mathcal{H}_{n}, f \in L^{2}(d q)\right)
$$

where $C(a, q)$ lies in the unitary group of $\mathcal{H}_{n}$ for almost all $q$. The cocycle property of $C$ is expressed by the identity

$$
C\left(a_{1}+a_{2}, a_{3}\right)=C\left(a_{1}, a_{3}\right) C\left(a_{2}, a_{1}+a_{3}\right) .
$$

Taking $a_{1}=q, a_{2}=a, a_{3}=0, b(q)=C(q, 0)$ we get

$$
C(a, q)=b(q)^{-1} b(a+q) \text {. }
$$


If we write $T$ for the automorphism

$$
T: g \otimes f(q) \longmapsto b(q) g \otimes f(q)
$$

then

$$
T^{-1} U^{0}(a) T=U(a) .
$$

The irreducibility then forces $n=1$ and we get the uniqueness of the Schrödinger representation. In his paper Mackey essentially gave this proof but with meticulous attention paid to the measure theoretic subtleties.

We have already remarked that this is really a story of a pair $(U, P)$ defined on $G$ satisfying (**), where $P$ is a projection valued measure on $G$ and $U$ is a unitary representation of $G$. He almost immediately realized that $P$ need only be defined on a space $X$ which has a $G$-action to make sense of $(* *)$. He thus obtained what one may now call a representation of a $G$-space $X$, namely, a pair $(U, P)$ where $P$ is a projection valued measure on a second countable locally compact space $X$ on which $G$ acts continuously, $G$ being a second countable locally compact group, and $U$ is a unitary representation of $G$, everything on a Hilbert space $\mathcal{H}$, satisfying the relation

$$
U(g) P_{E} U(g)^{-1}=P_{g[E]} \quad(g \in G, E \text { a Borel set } \subset X) .
$$

The previous case is obtained when $X=G$ with $G$ abelian and acting by translations. This generalization proved very fortuitous as well as fertile for him and completely determined his entire scientific thrust from then onwards. The pairs $(U, P)$ generalize the systems of imprimitivity first introduced by Frobenius, and so we shall call them (as Mackey did) systems of imprimitivity also. Like Frobenius, Mackey looked at the case when the action of $G$ on $X$ was transitive, but not necessarily simply transitive as in the previous setting, and obtained the complete generalization of the classical Frobenius theorem, what is known as the imprimitivity theorem [Ma6].

If the action of $G$ is not transitive, the orbit space $G \backslash X$ enters the picture, and Mackey's theory would lead to a complete description when the orbit space is what he would later call smooth, but in the contrary case ergodic phenomena would appear, introducing entirely new themes that were nonclassical.

When the action of $G$ on $X$ is transitive but not necessarily simply transitive, the stabilizers of points on $X$ are non trivial subgroups of $G$. Let $x_{0} \in X$ be arbitrary and let $H$ be the stability subgroup of $G$ at the point $x_{0}$, so that, when $G$ acts transitively (as we shall assume from now on), we have the isomorphism of locally compact spaces $G / H \simeq X$ given by $g H \longmapsto g\left[x_{0}\right]$. Then for any unitary representation $\sigma$ of $H$ one can associate a system of imprimitivity $\left(U^{\sigma}, P^{\sigma}\right)$ which can be viewed as a unitary representation of the pair $(G, X)$, and the basic theorem is that the correspondence

$$
F M: \sigma \longmapsto\left(U^{\sigma}, P^{\sigma}\right)
$$

is an equivalence of categories, from the category of unitary representations of $H$ to the category of unitary representations of the pair $(G, X)$. We shall call $F M$ the Frobenius-Mackey functor.

To explain what a great innovating leap it was to go from Weyl-Stone-von Neumann to Frobenius-Mackey, let me consider the special differential geometric subcategory where $G$ is a Lie group, $X$ is a smooth manifold on which the action 
of $G$ is smooth, and $\sigma$ is finite dimensional. Let us also assume that there is a $G$-invariant Borel measure $\mu$ on $X$. Then we have a functor

$$
V \longmapsto \mathcal{B}_{V}
$$

from the category of finite dimensional (left) $H$-modules $V$ to the category of vector bundles on $X$ with a $G$-action (these are the ones for which the pull back bundles on $G$ become trivial), and one knows that this is an equivalence of categories. If we write $\mathcal{D}_{V}$ for the space of smooth sections of $\mathcal{B}_{V}$ with compact support, then $G$ acts on $\mathcal{D}_{V}$. If we now assume that $V$ is unitary with $\sigma$ denoting the corresponding unitary representation of $H$, fiber-wise integration using $\mu$ will give a $G$-invariant scalar product on $\mathcal{D}_{V}$ whose completion $\mathcal{H}_{V}$ will be a Hilbert space, namely the Hilbert space $L^{2}\left(\mathcal{B}_{V}\right)$ of square integrable sections of $\mathcal{B}_{V}$, on which $G$ acts via the natural action on sections giving a unitary representation $U^{\sigma}$, and $P^{\sigma}$ is defined by $P_{E}^{\sigma}: s \longmapsto \chi_{E} s\left(s \in L^{2}\left(\mathcal{B}_{V}\right)\right)$.

Mackey's theory went much farther than this differential geometric special case. In the first place no smoothness is assumed, and the $\sigma$ can be infinite dimensional, although it must be admitted that in the differential geometric setting unitarity is not natural and one may allow $\sigma$ to be non unitary. Mackey constructed a Hilbert space bundle $\mathcal{B}_{V}$ associated to the unitary $V$ with $U^{\sigma}$ as the action on the sections of this bundle and $P^{\sigma}$ as above. It must be remarked that in the general locally compact case the bundles are not necessarily locally trivial and one has to operate in the Borel category as Mackey did. However at the time he did this work he finessed this issue by going over to $G$ in place of $X$ where the pull back bundles are trivialized and so the Hilbert space of sections becomes the subspace of $L^{2}(G, V)$ satisfying

$$
f(g \xi)=\sigma(\xi)^{-1} f(g) \quad(g \in G, \xi \in H),
$$

and $G$ acts by left translations. One has to make a modification if $X$ has no $G$ invariant measure. However there is always a unique G-invariant measure class and one may use any measure $\mu$ in this class and compensate for its lack of invariance by introducing the factors

$$
\rho_{g}=\left\{\frac{d \mu_{g}}{d \mu}\right\}^{1 / 2} .
$$

This amounts to tensoring by a line bundle. The representation $U^{\sigma}$ is the representation of $G$ induced by the representation $\sigma$ of $H$,

$$
U^{\sigma}=\operatorname{Ind}_{H}^{G} \sigma .
$$

Of course $P^{\sigma}$ is the usual projection valued measure with $P_{E}^{\sigma}$ as multiplication by $\chi_{E}$. The final definitions are, with $s$ a square integrable section,

$$
U^{\sigma}(g) s(x)=\rho_{g}\left(g^{-1}[x]\right) g \cdot s\left(g^{-1}[x]\right), \quad P_{E}^{\sigma} s(x)=\chi_{E}(x) s(x) .
$$

Thus the Frobenius-Mackey functor is defined on the category of pairs $(G, H)$ with $G$ a separable locally compact group and $H$ a closed subgroup. He worked out his theory of induced representations in this generality in two papers in the Annals [Ma7] [Ma8].

In his Seattle lectures he asserted that a very large part of representation theory can be brought under the theme of induced representations, and it was clear when talking to him that he was legitimately proud of his role and contributions to this theory. Of course it required the genius of Gel'fand and Harish-Chandra to see that for semi simple $G$, induction from a parabolic subgroup is the key to producing 
irreducible unitary representations. Eventually it became clear that this method, with suitable modifications (holomorphic induction, infinitesimal induction, cohomological induction, etc) will give most of the irreducible unitary representations for semi simple $G$. This program took a considerable amount of time and could not even be begun till after Harish-Chandra, in his monumental series of papers, constructed the discrete series. Nevertheless, it is really remarkable that at such an early stage of unitary representation theory one had the foundations for a complete theory of induced representations in the full locally compact category, due to Mackey's work. One must remember that Mackey worked out his theory long before the work of Chevalley, Gel'fand, Harish-Chandra, and Weil had shown the importance of doing the representation theory of Lie groups over $p$-adic fields and adele rings, and very few had a clear vision of where representation theory of non abelian non compact groups was going. When we consider how important the concepts of systems of imprimitivity and the imprimitivity theorem have turned out to be in recent generalizations such as the theory of unitary representations of super Lie groups and the $p$-adic representation theory of groups, one should say that his feeling of pride and achievement were well justified, perhaps even too modest.

In the summer of 1962 the International Congress of Mathematicians met in Stockholm, Sweden. Among the plenary speakers were Selberg and Gel'fand, and their lectures addressed questions largely concerned with the decompositions into irreducible pieces of induced representations of semi simple Lie groups. Gel'fand had been denied permission by the Soviet authorities to attend the conference, and Mackey delivered his address instead; if I remember correctly, Mackey mentioned in his speech that Gel'fand had sent the manuscript through his student Kirillov. I attended this Congress and therefore had a great opportunity to observe how central the themes of Mackey were in representation theory.

\section{Semi direct products and the little group method. Representations of finite length and orbit schemes}

Already (see [Ma6]) at the time of his creation of the theory of induced representations for the full category of locally compact separable groups in the early 1950's, Mackey considered its application to the description of all irreducible unitary representations of semi direct products $G=A \times^{\prime} H$ where $A$ is abelian and $H$ acts on $A$ as a group of automorphisms via $h, a \longmapsto h[a] ; H$ is not necessarily abelian. The group law is

$$
\left(a_{1}, h_{1}\right)\left(a_{2}, h_{2}\right)=\left(a_{1}+h_{1}\left[a_{2}\right], h_{1} h_{2}\right) .
$$

The prototype of this type of group is the Poincaré group, important in relativistic quantum theory, where $A=\mathbf{R}^{4}$ and $H$ is the connected Lorentz group or rather its simply connected two-fold cover isomorphic to $\operatorname{SL}(2, \mathbf{C})_{\mathbf{R}}$ where the suffix $\mathbf{R}$ means that it is a question of the real group underlying the complex group. The unitary representations of $G$ can be viewed as a pair of unitary representations $U, V$ of $A, H$ respectively, with

$$
V(h) U(a) V(h)^{-1}=U(h[a]) \quad(a \in A, h \in H) .
$$

If we write

$$
U(a)=\int_{\widehat{A}} \xi(a) d P(\xi)
$$


where $P$ is the spectral measure of $U$, the above relation between $U$ and $V$ becomes

$$
V(h) P_{E} V(h)^{-1}=P_{h[E]} \quad(h \in H, E \text { a Borel set } \subset \widehat{A})
$$

and $h[E]$ is the transform of $E$ under the action of $H$ on $\widehat{A}$ dual to the given action of $H$ on $A$. This is just a system of imprimitivity for the pair $(H, \widehat{A})$ but the action of $H$ on $\widehat{A}$ may not be transitive. If we assume that the original representation is irreducible, then one can prove that $P_{E}=0$ or $I$ for any invariant Borel set $E$. This last property will be true if $P$ is supported by an orbit, but experience with ergodic theory shows that this need not always be the case. Mackey realized that if there is a Borel cross section for the orbit space $H \backslash \widehat{A}$, then all such $P$ are supported on orbits. $P$ is then essentially transitive, and the theory applies. This led to his famous theorem that the irreducible unitary representations are parametrized by $(m, \sigma)$ where the $m$ are points of a Borel set meeting each orbit exactly once, and $\sigma$ is an irreducible unitary representation of the stabilizer $H_{m}$ of $m$. For the Poincaré group this leads at once to the classification of elementary particles by their mass and spin. In this special case this was already carried out by essentially the same method by Wigner in 1939 [Wi]; the stabilizers are known to the physicists as little groups ever since.

When $H \backslash \widehat{A}$ has a Borel cross section, we say that the semi direct product is regular. There are semi direct products which are not regular, and these typically involve some ergodicity brought about by arithmetical aspects. Regularity is the norm in most applications. Verification of regularity appeared to be a global question till the work of Effros $[\mathbf{E}]$ and Glimm $[\mathbf{G l}]$ showed that it is really local. More precisely, for a pair $(G, X)$ in the locally compact second countable category, $G \backslash X$ has a Borel cross section if and only if every $G$-orbit is locally closed in $X$, and that this is the same as requiring that the Borel structure on $G \backslash X$ is standard, or even countably separated.

Of the same genre is the strong suggestion made by Mackey in his Chicago lectures that a separable locally compact group is of type I if and only if it has a smooth dual (see below). This was proved by Glimm [Gl].

Representations of group extensions. In the case $G=A \times^{\prime} H$ above, $A$ is normal in $G$, and it is natural to ask if one can treat by similar methods the more general case where we have a group $G$ with an abelian normal subgroup $A$, but do not assume that $G$ splits as a semi direct product. The objective is then to reduce the representation theory of $G$ to those of $A$ and the subgroups of $H=G / A$. In this formulation there is no need to assume that $A$ is abelian. Mackey undertook a study of this question and the results appeared in his paper [Ma9] although most of them must have been obtained much earlier, since there is a discussion of them already in [Ma2]. He assumed that $A$ is of type $\mathrm{I}$ in the sense of the theory of von Neumann algebras and that its unitary dual $\widehat{A}$ is smooth, i.e., $\widehat{A}$ is a standard Borel space; actually these properties are equivalent, as was later shown by Glimm [Gl]. The action of $G$ on itself by inner automorphisms gives an action on $A$ and hence on $\widehat{A}$, and it is clear that $A$ acts trivially so that we really have an action of $H=G / A$ on $\widehat{A}$. If this action is regular in the sense that $H \backslash \widehat{A}$ is a standard Borel space, then the semi direct product theory can be extended with an important modification: if $m \in \widehat{A}$ and $H_{m}$ is the stabilizer of $m$ in $H$, one requires the knowledge of projective representations of $H_{m}$ to construct the unitary 
representations of $G$. I shall discuss projective representations a little later. But, using the notation therein, the multipliers of the projective representations of $H_{m}$ are determined uniquely. While this does not give the inductive mechanism we need, it turns out that the projective representations with a multiplier $\sigma$ of $G$ still reduce only to orbits of $H$ in the $\sigma$-dual of $A$ and projective $\tau$-representations of $H_{m}$ ( $m$ a fixed element in the $\sigma$-dual of $A$ ) where $\tau$ is determined by $\sigma$ canonically. It is thus clear that we have an inductive mechanism for understanding the representation theory of $G$ in terms of those of $A$ and $H=G / A$.

This procedure, known as the Mackey machine, does indeed succeed in certain nilpotent groups whose central sequences are of small length, but the general case becomes very cumbersome. However, very shortly afterwards, Kirillov developed a beautiful direct method of inducing by one dimensional representations of suitable subgroups, to obtain the representation theory of all nilpotent Lie groups. The architecture of a definitive theory of unitary representations of solvable groups became clear with the works of Auslander-Moore, Auslander-Kostant, Pukanszky, and a host of others. However all these works are in the context of Lie groups, and Mackey's dream of doing things in the full locally compact category seems to have been forgotten.

Representations of finite length and orbit schemes. In covariant (GuptaBleuler) quantum electrodynamics one couples the Maxwell field with the Dirac field, and for this purpose it is very useful to realize the photon representation as a subquotient of a non unitary but natural representation associated to the zero mass orbit of the Lorentz group. This representation was imbedded in a family of deformations by G. Rideau $[\mathbf{R}]$. On the other hand, in his studies of gravity, Nakanishi $[\mathbf{N}]$ introduced a family of representations of the Poincaré group which acted on functions defined on the light cone but the action depending on the derivatives of these functions in transverse directions to the light cone. In [CTV] this situation was examined carefully and representations were constructed on the sections of the 1-jet bundle on the light cone, the Poincaré action being completely natural, thus explaining the procedure of Nakanishi as well as describing the Rideau representations in a canonical manner. This suggested that there is a theory of representations of the Poincaré group which are obtained by its natural action on the jet bundles on the light cone. More generally, if we have a regular semi direct product $G=A \times^{\prime} H$ where $A$ is a vector group and $H$ a closed subgroup of $\operatorname{GL}(A)$, one could ask whether the Mackey correspondence between irreducible representations of $G$ and orbits in $\widehat{A}$ can be extended to a correspondence between representations of finite length of $G$ whose irreducible constituents are associated to an orbit $O$, and representations arising out of the natural action of $G$ on sections of vector bundles defined on infinitesimal neighborhoods of $O$ (orbit schemes.). This problem was studied in great depth by A. Guichardet [Gui1] [Gui2], his student du Cloux [du C], and later, by my student Charles H. Conley in $[\mathbf{C H C 1}][$ CHC2]. Their work has yielded many beautiful results in this context. In particular Guichardet showed that the Rideau phenomenon can arise only if the tangent space to the orbit (at a point of it) does not admit an invariant complement (as in the case of the light cone), and completely determined what happens in the other, complemented case, while du Cloux studied the nilpotent case. Conley proved that when $H$ is algebraic and the stabilizer at a point of $O$ has the property that all its finite dimensional representations are rational, then all representations of length $n+1$ whose irreducible 
constituents arise from the orbit $O$ via the Mackey correspondence, can be obtained as subquotients of the actions of $G$ on sections of vector bundles defined on the $n^{\text {th }}$ order infinitesimal neighborhood of $O$. It is appropriate to draw attention to these papers and suggest that these issues be examined again in other directions.

Weyl commutation relations and quantum information theory. The Weyl-Mackey commutation rules for finite abelian groups have an interpretation in quantum information theory which I shall now briefly indicate. Quantum information theory is based on the idea that information can be stored or encoded as quantum states in a finite dimensional complex Hilbert space $\mathcal{H}$ transmitted through a channel and retrieved by generalized measurements. During transmission quantum states can be corrupted by noisecharacterized by a linear subspace $\mathcal{E}$ in the algebra $\mathcal{B}(\mathcal{H})$ of all operators on $\mathcal{H}$. If $\rho$ is the input state of the channel then the corrupted output state $T(\rho)$ is of the form

$$
T(\rho)=\frac{1}{\operatorname{Tr} \rho \sum_{\mathrm{i}} \mathrm{E}_{\mathrm{i}}^{\dagger} \mathrm{E}_{\mathrm{i}}} \sum_{i} E_{i} \rho E_{i}^{\dagger}
$$

where the corrupting operators $\left\{E_{i}\right\}$ constitute an arbitrary finite subset of $\mathcal{E}$. One does not have a knowledge of the specific subset $\left\{E_{i}\right\}$ occurring above. To such a corrupted output state one can apply a recovery or decoding operation $\mathcal{R}$ so that the filtered output state is

$$
\mathcal{R}(T(\rho))=\sum_{j} R_{j} T(\rho) R_{j}^{\dagger}
$$

where $\sum_{j} R_{j}^{\dagger} R_{j}=I$. Given $\mathcal{E}$, the aim of the theory of error-correcting quantum codes is to construct a pair $(\mathcal{C}, \mathcal{R})$ where $\mathcal{C}$ is a 'large' subspace of $\mathcal{H}$ and $\mathcal{R}$ is a decoding operation with the property that, for every state $\rho$ with support in $\mathcal{E}$,

$$
\mathcal{R}(T(\rho))=\rho .
$$

The subspace $\mathcal{C}$ occurring here is called a quantum error-correcting code for $\mathcal{E}$ with size $=\operatorname{dim} \mathcal{C}$. The construction of such a code $\mathcal{C}$ is equivalent to the construction of the corresponding projection $P$ on $\mathcal{C}$. Such a projection $P$ is expressed as the average of a finite abelian group of unitary operators on $\mathcal{H}$ coming from the projective representation of $A \oplus \widehat{A}$ :

$$
P=\frac{1}{|H|} \sum_{(a, b) \in H} \sigma(a, b) U_{a} V_{b}
$$

where $H$ is a subgroup of $A \oplus A$ and the map $(a, b) \rightarrow \sigma(a, b) U_{a} V_{b}$ is a unitary representation of $H,(U, V)$ being the pair of unitary representations of $A$ occurring in the Weyl-Mackey theory (with $\widehat{A} \simeq A$ via a non-degenerate bicharacter of $A$ ). For a good introduction to the ideas outlined above we refer to the book of Nielsen and Chuang $[\mathbf{N C}]$. The reader may also see $[\mathbf{P 1}]$.

\section{Super Lie groups and their systems of imprimitivity}

In the 1970's, propelled by the urge to create a fully unified theory of all the fundamental forces, the physicists discovered super symmetry. Super symmetry is a generalization of the usual concept of symmetry and plays an important and very essential role in creating a unified theory of matter and radiation. Mathematically 
one needs a generalization of ordinary differential geometry, namely super geometry, to define clearly what is meant by a super symmetry. A super manifold is a topological space $M$ with a structure sheaf $\mathcal{O}$ consisting of $\mathbf{Z}_{2}$-graded algebras that is locally of the form $C^{\infty}\left(x_{1}, x_{2}, \ldots, x_{p}\right)\left[\theta_{1}, \theta_{2}, \ldots, \theta_{q}\right]$, the $\theta_{j}$ being Grassmann variables, i.e., satisfy

$$
\theta_{i} \theta_{j}+\theta_{j} \theta_{i}=0
$$

It is important to note that when the odd variables (which cannot be seen) are made 0 , the sheaf reduces to that of the classical manifold $M$ which is a submanifold of the super manifold. Super manifolds are thus objects similar to the schemes of Grothendieck, and are studied by similar techniques. Super symmetries are automorphisms of super manifolds. The simplest example is $\mathbf{R}^{1 \mid 2}$ with $\mathcal{O}(U)=$ $C^{\infty}(U)\left[\theta_{1}, \theta_{2}\right]$. The automorphism defined by

$$
t \longmapsto t+\theta_{1} \theta_{2}, \quad \theta_{i} \longmapsto \theta_{i}(i=1,2)
$$

is a typical super symmetry. A super Lie group is a group object in the category of super manifolds. It can also be viewed as a group-valued functor on the category of super manifolds. All the fundamental results of classical Lie theory extend to the super Lie groups (with suitable modifications) and there is now a well understood theory of super Lie groups and their super homogeneous spaces [Va1]. In particular the physicists have constructed the super Poincaré groups as super symmetric extensions of the classical Poincaré groups (in arbitrary dimension), and it is natural to ask if one can classify the unitary representations of the super Poincaré groups in a manner similar to the classical case. It must be mentioned that because a super Lie group is not a group but a group-valued functor, the concept of its unitary representations is more subtle to define, but this can be done. Indeed, a super Lie group $G$ gives rise to the underlying classical Lie group $G_{0}$ as well as to a super Lie algebra $\mathfrak{g}$, with an action of $G_{0}$ on $\mathfrak{g}$; such a pair is called a Harish-Chandra pair. The assignment $G \longmapsto\left(G_{0}, \mathfrak{g}\right)$ is an equivalence of categories from the category of super Lie groups to the category of Harish-Chandra pairs. We may therefore define a unitary representation $\pi$ of $G$ as a pair $\left(\pi_{0}, \rho\right)$ where $\pi_{0}$ is a unitary representation of $G_{0}$ and $\rho$ a representation of $\mathfrak{g}$, with suitable covariance conditions. Now the operators of $\rho$ are typically unbounded but one can prove the fundamental theorem that they are essentially self adjoint and that therefore there is a uniqueness in their definition. One can then prove the imprimitivity theorem for a super homogeneous space $G / H$ where $G$ is a super Lie group and $H$ is a closed super Lie subgroup, at least when $X=G / H$ is purely even, i.e., a classical manifold. This leads as in the classical theory to a complete description of irreducible unitary representations of super semi direct products (not just the super Poincaré groups) $A \times^{\prime} H$ ( $A$ is a super abelian group) which are regular at the classical level (when all odd coordinates are put to 0) [CCTV1]. In turn this leads to the classification of super particles and the elucidation of the concept of super multiplets. The physicists had already worked out the classification of super particles and super multiplets in the 1970's, but their methods assumed as an article of faith that the method of little groups could be extended to the super context; moreover these results were confined to the super Poincaré groups and did not touch the full semi direct products in the super category, nor did they treat the case of infinite spin in the massless case for the super Poincaré groups. It must also be mentioned that whereas all orbits of $\widehat{A}$ 
contribute representations classically, in the super symmetric case only those with positive energy appear.

The restriction that $G / H$ is purely even is just for convenience. The theory can be extended to the general case [CCTV2].

\section{Intertwining numbers, double coset decompositions, and irreducibility of induced representations}

Let $G, H$ be as above. Then we have the functor of induced representations

$$
\sigma \longmapsto U^{\sigma}=\operatorname{Ind}_{H}^{G} \sigma .
$$

We have omitted $P^{\sigma}$ and so this can no longer be expected to be an equivalence of categories. In particular

$$
\operatorname{Hom}\left(U^{\sigma_{1}}, U^{\sigma_{2}}\right)
$$

will in general be much bigger than

$$
\operatorname{Hom}\left(\sigma_{1}, \sigma_{2}\right)
$$

and it is clearly crucial to determine

$$
\operatorname{Hom}\left(U^{\sigma_{1}}, U^{\sigma_{2}}\right) \text {. }
$$

More generally, if $H_{i}(i=1,2)$ are two closed subgroups and $\sigma_{i}$ is a unitary representation of $H_{i}$, then one is interested in determining

The condition

$$
\operatorname{Hom}\left(\operatorname{Ind}_{H_{1}}^{G} \sigma_{1}, \operatorname{Ind}_{H_{2}}^{G} \sigma_{2}\right) \text {. }
$$

$$
\operatorname{Hom}\left(\operatorname{Ind}_{H}^{G} \sigma, \operatorname{Ind}_{H}^{G} \sigma\right)=\mathbf{C}
$$

is necessary and sufficient for the irreducibility of the induced representation.

The structure of the induced representations for finite groups became important in the 1930's in Artin's work on $L$-functions associated to Galois representations, especially the question of how the general representation may be constructed in terms of representations induced from one dimensional representations (monomial representations). However not every irreducible representation of $G$ is monomial. In order to prove that the Artin non abelian $L$-functions are meromorphic over the whole $s$-plane it would be enough to prove that every irreducible representation of $G$ is at least a virtual linear combination

$$
\sum_{i} n_{i} \operatorname{Ind}_{H_{i}}^{G} \sigma_{i}
$$

where the $n_{i}$ are integers which are not necessarily positive, and the $\sigma_{i}$ are one dimensional. This is what Brauer did.

Mackey's approach to the determination of

$$
\operatorname{Hom}\left(\operatorname{Ind}_{H_{1}}^{G} \sigma_{1}, \operatorname{Ind}_{H_{2}} \sigma_{2}\right)
$$

was very direct. When $G$ is finite the elements of the Hom space, called intertwining operators by him following a suggestion of Kneser, may be identified with linear operator valued functions on $G$ with suitable transformation properties under translations from left by $H_{1}$ and right by $H_{2}$. Hence they are determined by the double coset space

$$
H_{1} \backslash G / H_{2} \text {. }
$$

Using this Mackey [Ma10] determined the dimension of the Hom space, the socalled intertwining number $I$, as a sum over the double coset space of intertwining 
numbers associated to pairs of representations corresponding to the elements of a general double coset. For $x, y \in G$ let $I(x, y)$ denote the intertwining number of the representations $s \longmapsto \sigma_{1}\left(x s x^{-1}\right), s \longmapsto \sigma_{2}\left(y s y^{-1}\right)$ of the subgroup $x^{-1} H_{1} x \cap$ $y^{-1} H_{2} y$. Then $I(x, y)$ depends only on the double coset $D=H_{1} x y^{-1} H_{2}$ and so we may write it as $I(D)$. If $\mathcal{D}$ is the space of double cosets $H_{1} \backslash G / H_{2}$, then

$$
I=\sum_{D \in \mathcal{D}} I(D)
$$

In particular, if $H_{1}=H_{2}=H$ and $\sigma_{1}=\sigma_{2}=\sigma$, this formula yields the following sufficient condition for the irreducibility of $\operatorname{Ind}_{H}^{G} \sigma$. For any $x \in G$ let $\sigma_{x}$ be the representation $s \longmapsto \sigma\left(x s x^{-1}\right)$ of $x^{-1} H x$, and let us suppose that for each $x \notin H$ the restrictions to $x^{-1} H x \cap H$ of the representations $\sigma_{x}, \sigma$ have no irreducible in common; then $\operatorname{Ind}_{H}^{G} \sigma$ is irreducible. These results of Mackey included all known results on induced representations for finite groups, and more importantly, set the stage for determining the Hom space and intertwining numbers when $G$ is no longer assumed to be finite.

When $G$ is arbitrary, the spaces on which the induced representations act are Hilbert spaces, and the linear operators between them have a more complicated structure than in the finite case. So the method of Mackey has to be supplemented by a systematic use of analysis of linear operators between function spaces. At that time Schwartz had just published his epoch-making books on distribution theory and had proved a theorem (kernel theorem) from which it followed that general linear maps between function spaces on manifolds $X_{i}(i=1,2)$ can be represented by distributions on $X_{1} \times X_{2}$. Bruhat, who was in Paris as a student and was interacting with Schwartz, realized that the kernel theorem of Schwartz offered the exact tool to carry out the Mackey analysis when $G$ is a Lie group. Using the theory of distributions he determined the Hom space by an almost exact analogue of Mackey's formula in the case when $G$ is a connected semi simple real Lie group, $H=P$ is a minimal parabolic subgroup, and $\sigma$ is a finite dimensional irreducible unitary representation of $H$ trivial on the unipotent radical of $H$ (the so-called principal series representations). The double coset space $P \backslash G / P$ is now finite, an instance of the famous Bruhat decomposition, and Bruhat's variant of the Makey criterion becomes the following sufficient condition: if for all elements $w$ of the relative Weyl group with $w \neq 1$, the transform $\sigma^{w}$ is not equivalent to $\sigma$, then $\operatorname{Ind}_{P}^{G} \sigma$ is irreducible. Bruhat's debt to Mackey is very clear. Bruhat subsequently extended his work to include the principal series for $p$-adic semi simple Lie groups [Br1] [Br2].

At the risk of some digression I shall try to give a partial explanation of the remarkable fact that in spite of the huge technical difference between the finite and Lie theoretic cases, the criterion for irreducibility of the induced representation (in the context of parabolic induction for semi simple groups) takes essentially the same form. The situation is the following. We have a Lie group $H$ acting on a manifold $X$ and we are interested in determining, for a given $H$-module $E$, all the invariant $E$-distributions. There are two cases: when there are finitely or countably many orbits, and when there are "moduli" for the orbits; only the first case occurs for the irreducibility problem and there the orbit space is finite. The filtration by dimension of the orbit space gives rise to a filtration on the space of invariant $E$-distributions, and the corresponding graded spaces are (roughly) the spaces of invariant $E$-distributions supported by a given orbit $O \subset X$. Unlike what happens in 
the finite or the $p$-adic case, the transversal differentiations can in principle produce invariant distributions supported by and not living on the orbit $O$. The key now is to replace the $E$-distributions $T$ supported by $O$ to the $F \otimes E$-distributions $\sigma(T)$ that live on $O, \sigma(T)$ being the transverse symbol of $T, F$ being the dual of the transverse jet bundle of $O$ in $X$. Because we are now dealing with distributions living on $O$, the transitivity of $H$ on $O$ now allows the space of $F \otimes E$-distributions to be identified with the space of invariants of the fiber at at a single point on the orbit $O$ which is determined by a simple calculation. The fiber modules however are very different in the higher transversal directions from the module on $E$ (roughly speaking, $E$ is pure imaginary while $F$, being geometric, is real); and this forces the conclusion that no transversal differentiation can produce invariants. In this manner transversal invariants are ruled out which gives the result. For details see $[\mathrm{KV}]$.

One final personal remark may not be out of order. I first heard of Bruhat's theorem on the irreducibility of the principal series from Mackey's 1961 Seattle lectures and the idea came to me at that time that one could attack it infinitesimally. I went to India in 1962 and started to work on the infinitesimal theory, first with Varadhan, and after he left for the Courant Institute, with Ranga Rao and Parthasarathy. We obtained the full criterion of irreducibility for all spherical principal series (unitary or not) for complex semi simple groups and published it in $[\mathbf{P R V}]$. It was my first paper on semi simple Lie groups and its genesis can directly be traced to the inspiration I received from Mackey's lectures. The problem of irreducibility from the infinitesimal point of view has since been taken to great heights-by Wallach for the complex groups (not just spherical series alone as in our work), by Kostant-Rallis and Kostant for spherical series for real groups, by Enright-Wallach for the fundamental series, and so on.

If $G$ is a semi simple group, its representations come in various series, following the terminology first invented by Gel'fand and Naimark. Harish-Chandra eventually succeeded in proving that the induced representations corresponding to the various Cartan subgroups have finite length and generically irreducible. Later on he obtained a more general irreducibility theorem where the parabolic subgroup from which the induction is made is arbitrary, and the inducing representation has a real infinitesimal character (real in a suitable sense). To prove this he used a variant of Bruhat's method [Ha1]. For a discussion of this question see again [KV].

One should not assume that the finite case is just an exercise. The theory of unitary representations of semi simple groups defined over a finite field requires very deep applications of algebraic geometric methods. For an interesting view see [Ha2]. See also the article of Vogan in this volume.

\section{Projective representations, Borel cohomology of groups, and the metaplectic representation}

If $G$ is a standard Borel group, a projective representation of $G$ is a Borel map into the projective unitary group $\mathrm{PU}(\mathcal{H})$ of a separable Hilbert space $\mathcal{H}$. Since there is a Borel cross section for the map $\mathrm{U}(\mathcal{H}) \longrightarrow \mathrm{PU}(\mathcal{H})$ it can also be thought of as a Borel map $L$ of $G$ into $\mathrm{U}(\mathcal{H})$ such that

$$
L(x y)=m(x, y) L(x) L(y) \quad(x, y \in G), \quad L(1)=1 .
$$


Here $m$ is a Borel map of $G \times G$ into $S^{1}$ satisfying the following identities derived from the associativity of the group multiplication:

(a) $m(x, 1)=m(1, x)=1(x \in G)$

(b) $m(x y, z) m(x, y)=m(x, y z) m(y, z)(x, y, z \in G)$.

It is called the multiplier of $L$. The set of all functions(multipliers) satisfying these identities form an abelian group $C(G)$. It can be shown that any element of $C(G)$ can be the multiplier of a projective unitary representation of $G$ if $G$ is separable locally compact; if $m$ is the multiplier, representations with multiplier $m$ are called $m$-representations. Changing $L$ to $L^{\prime}=a L$ where $a$ is a Borel map of $G$ into $S^{1}$ with $a(1)=1$ results in the change

$$
m(x, y) \longmapsto m^{\prime}(x, y)=m(x, y) \frac{a(x y)}{a(x) a(y)} .
$$

We say $m$ and $m^{\prime}$ are equivalent. The $m$ that are equivalent to 1 are called trivial and form a subgroup $B(G)$ of $C(G)$. The quotient group $M(G)=C(G) / B(G)$ is called the multiplier group of $G$. It may be regarded as a Borel version of the cohomology group $H^{2}\left(G, S^{1}\right)$, with the multipliers being just the Borel measurable cocycles.

Projective representations go back a long time. Already Schur had determined the projective representations of the symmetric and alternating groups. Then Weyl encountered them in his fundamental work on quantum kinematics. As we have seen earlier, he expressed the equations of quantum kinematics as a pair of unitary representations $U, V$ of abelian groups $A, B$ in duality by a bicharacter $\chi$ such that

$$
U(a) V(b)=\chi(a, b) V(b) U(a) \quad(a \in A, b \in B) .
$$

He then regarded

$$
(a, b) \longmapsto U(a) V(b)
$$

as a projective representation of the abelian group $A \times B$, and formulated quantum kinematics as the additional structure provided by a projective representation of $A \times B$ which in applications is the affine phase space $P=A \oplus A$ where $A$ is a finite dimensional real euclidean space, the affine configuration space. Actually, if we use von Neumann's refinement, we can define

$$
W(a, b)=e^{-a \cdot b / 2} U(a) V(b) \quad(a, b \in A)
$$

where $a \cdot b$ is the scalar product of $a$ and $b$, then $W$ is a projective representation of $P$ with multiplier

$$
\sigma\left((a, b),\left(a^{\prime}, b^{\prime}\right)=e^{i\left(a^{\prime} \cdot b-a \cdot b^{\prime}\right) / 2}=e^{i \beta\left((a, b),\left(a^{\prime}, b^{\prime}\right)\right) / 2}\right.
$$

where

$$
\beta\left((a, b),\left(a^{\prime}, b^{\prime}\right)\right)=a^{\prime} \cdot b-a \cdot b^{\prime}
$$

is the natural symplectic structure on $P$. The uniqueness of Schrödinger representation is the statement that $P$ has a exactly one (up to equivalence) irreducible $\sigma$-representation, namely the Schrödinger representation, and that every representation is a multiple of this.

The Weyl-Wigner point of view asserts that the covariance of a quantum system with respect to a symmetry group $G$ is expressed by the specification of a projective unitary representation of $G$ in the quantum Hilbert space, and so it is appropriate that quantum kinematics is described by the essentially unique irreducible projective representation of the phase space (it can be shown that multipliers of $P$ are of 
the form $e^{i \gamma}$ where $\gamma$ is a skew symmetric bilinear form on $A \times A$, and so generically reduce to the one given above). Later on, Wigner proved [Wi] that for the simply connected Poincaré group the multiplier group is trivial and so relativistic covariance is always expressed by ordinary representations of the Poincar group; the situation is very different for the Galilean group, as Bargmann showed in a seminal paper on projective representations of Lie groups [Ba].

The simply connected Galilean group is an example where simple connectivity does not ensure that all projective representations can be renormalized to be ordinary representations, in other words $H^{2}(G, T)$ can well be non trivial for simply connected $G$. For any given multiplier $m$ we have a central extension $H_{m}$ of $G$ by $T$,

$$
1 \longrightarrow T \longrightarrow H_{m} \longrightarrow G \longrightarrow 1
$$

such that $m$-representations of $G$ correspond one-one to ordinary representations of $H_{m}$ which map $t \in T$ to $t I$ for all $t$ (see below). However, as $m$-varies, the extension $H_{m}$ will vary, and it is natural to ask if there is a universal central extension that will work for all multipliers of $G$. Such a universal extension was constructed by Moore [Moo2]. If $G$ is a Lie group, which we may assume to be simply connected, a more explicit construction of the universal central extension $\bar{G}$ of $G$, which will also be a Lie group, can be found in [CDLL] where many examples are discussed. The group $\bar{G}$ is a central extension of $G$ :

$$
1 \longrightarrow K \longrightarrow \bar{G} \longrightarrow G \longrightarrow 1
$$

with

$$
K=H^{2}(G, \mathbf{R})=H^{2}(\operatorname{Lie}(G), \mathbf{R}) .
$$

(The last cohomology is a finite dimensional vector space.) The main result is that there is a natural bijection between physical equivalence classes of irreducible unitary representations of $\bar{G}$ that are scalars on $K$ and the physical equivalence classes of projective irreducible representations of $G$ (physical equivalence is coarser than unitary equivalence and requires only an equivalence implemented by an operator which is either unitary or antiunitary). The Galilean group in the $2+1$ dimensional case is important in the treatment of genuine two dimensional systems.

The existence and explicit construction of $\bar{G}$ allows us to replace symmetry actions of $G$ by actions via unitary representations of $\bar{G}$ which are scalars on $K$. It can be shown that for the Lie group $G$ with Lie algebra $\mathfrak{g}$, the condition $[\mathfrak{g}, \mathfrak{g}]=\mathfrak{g}$ is sufficient to ensure that $H^{2}(\bar{G}, T)=0$ [Di]. But it is not necessary; the result is true for the Galilean groups in space times of dimension $\geq 4$ which do not satisfy the above sufficient condition $[\mathbf{D e}]$.

Brauer groups and Borel cohomology. Mackey's theory of representations of group extensions led him to the notion of projective representations, and, for any multiplier $\sigma$, the $\sigma$-dual of a separable locally compact group, namely the set of equivalence classes of the irreducible $\sigma$-representations of the group in question. The multiplier group is a special case of the group $H^{2}(G, A)$ of $A$-valued multipliers where $A$ is an abelian separable locally compact group. Classically, when the groups are finite, the group $H^{2}(G, A)$ for arbitrary $G$ and abelian $A$ is an example of a Brauer group and its elements classify the central extensions of $G$ by $A$, namely the groups $H$ such that $A$ is central in $H$ and $H / A \simeq G$. Mackey discovered that this interpretation remains valid even in the locally compact category. The first point is that $H / A$ does not in general have continuous sections but always has Borel sections 
and so one can associate an element of $H^{2}(G, A)$ to the extension $H$; this shows the naturalness of working in Borel categories. In the reverse direction, given a $A$-valued multiplier $m$, the classical Brauer construction gives a group structure on $H_{m}=G \times A$ dependent on $m$ such that $H$ is an extension with multiplier $m$. But, since $m$ is only Borel, $H_{m}$ will not be locally compact in the product topology unless $m$ is continuous, which it seldom is. If $m$ is locally continuous near the identity, we can use the product topology near the identity of $G$ and then use translations by group elements to give the topology of the group; this will lead to the cases where $H$ is a locally trivial bundle over $H / A$ and thus take care of all Lie groups. In the general case this construction yields only a Borel group. But Mackey noticed that it has an invariant measure, namely the product of the Haar measures on $G$ and $A$. Now, Weil had shown that given an invariant measure on a group one can give it what we now call the Weil topology and the completion of the group in its Weil topology is locally compact. Mackey observed that when the group has an invariant measure and its Borel structure is standard, it is already locally compact in its Weil topology, thus realizing $H_{m}$ as a locally compact separable group. The construction of Brauer groups in the Borel category is one of his beautiful achievements [Ma11].

It became clear from Mackey's work that Borel cohomology of locally compact separable groups is something that should be explored systematically and in all degrees. His student Calvin re did precisely that in a series of papers; in particular he linked this theory with classfield theory of arithmetic fields [Moo1].

Metaplectic representation. Let us go back to the Weyl formulation of quantum kinematics for a configuration space $\mathbf{R}^{d}$ so that we have a pair of unitary representations of $U, V$ of $\mathbf{R}^{d}$ such that

$$
U(a) V(b)=e^{i a \cdot b} V(b) U(a) .
$$

So

$$
(a, b) \longmapsto W(a, b)=U(a) V(b)
$$

is a projective representation of $P=\mathbf{R}^{d} \oplus \mathbf{R}^{d}$ with multiplier $m$ where

$$
m\left((a, b),\left(a^{\prime}, b^{\prime}\right)\right)=e^{i a^{\prime} \cdot b} .
$$

If we now go to the equivalent projective representation

$$
(a, b) \longmapsto e^{-(i / 2) a \cdot b} W(a, b)
$$

the multiplier is changed to

$$
m^{\sim}\left((a, b),\left(a^{\prime}, b^{\prime}\right)\right)=e^{(i / 2)\left[a^{\prime} \cdot b-a \cdot b^{\prime}\right]} .
$$

Motivated by this we shall define for any separable locally compact abelian group $A$ the multiplier $\sigma$ for the group $G=A \times \widehat{A}$ by

$$
\sigma\left((a, \xi),\left(a^{\prime}, \xi^{\prime}\right)\right)=\frac{\left\langle a^{\prime}, \xi\right\rangle}{\left\langle a, \xi^{\prime}\right\rangle} .
$$

It is easy to verify that $\sigma$ induces a perfect pairing of $G \times \widehat{G}$ with itself and so it is natural to call the multiplier $\sigma$ symplectic; moreover any automorphism of the group $G$ that leaves $\sigma$ invariant will be called symplectic also. Let $\operatorname{Sp}(G)$ be the group of symplectic automorphisms of $G$. 
Suppose that $A$ has the property that $x \longmapsto 2 x$ is an automorphism of $A$; the same is then true for $\widehat{A}$ also and we say that $A$ and $\widehat{A}$ are 2-regular. Let $U, V$ be unitary representations of $A, \widehat{A}$ respectively such that

$$
U(a) V(\xi)=\langle a, \xi\rangle V(\xi) U(a)
$$

as in Mackey's work. We know that any such pair $U, V$ is a direct sum of copies of a unique irreducible pair. If we write $V^{2}(\xi)=V(2 \xi)$ it is then immediate that the same is true for the pair $U, V^{2}$ which now satisfy

$$
U(a) V^{2}(\xi)=\langle a, 2 \xi\rangle V^{2}(\xi) U(a) .
$$

Write now

$$
W(a, \xi)=\langle a, \xi\rangle^{-1} U(a) V^{2}(\xi) .
$$

Then $W$ is a projective representation of $G=A \oplus \widehat{A}$ with the multiplier $\sigma$ defined above, and $W$ gives back $U, V$ by $W(a, o)=U(a), W(0, \xi)=V^{2}(\xi)$. Thus we can say that when $A$, hence $G$ also, is 2-regular, every $\sigma$-representation of $G$ is a direct sum of copies of a unique irreducible one, which we can take in the standard form in $\mathcal{H}=L^{2}(A)$.

Mackey's generalization of the Stone-von Neumann uniqueness of the Schrödinger representation now shows that to any symplectic automorphism $s$ of $G$ there is a unitary operator $R(s)$ in $\mathcal{H}$, unique up to a phase factor, such that

$$
W(s(a, \xi))=R(s) W(a, \xi) R(s)^{-1} .
$$

The map $s \longmapsto R(s)$, because of the uniqueness of $R(s)$ up to a phase factor, determines a projective representation of $G$. In other words, associated to any 2-regular locally compact separable abelian group we have a canonical projective unitary representation of the symplectic group $\operatorname{Sp}(G)$ of $G=A \oplus \widehat{A}$ in $L^{2}(A)$.

The history of the discovery of this projective representation of the symplectic group is very interesting. It was discovered by Andre Weil, certainly in the case when $A$ is a vector space over a local field of finite dimension, or a free finite module over the adele ring of a global (arithmetic) field; he in fact credits the genesis of this representation to the work of Siegel on quadratic forms. In a pair of seminal papers, Weil discussed this representation and its role in arithmetic, casting light on the Siegel theory [We1] [We2]. In 1962, when he lectured in Harvard about these results, it was pointed out to him that at least when the local field was $\mathbf{R}$, the representation had been discovered independently by Irving Segal, and that Shale, a student of Segal, had then constructed this representation in the case when $A$ was not locally compact, in fact when it was the additive group of a real Hilbert space! [].

In a paper he published in 1965 [Ma12] Mackey treated these matters in complete generality. He showed that when $A$ is not 2-regular, new phenomena arise. Let $A^{2}=2 A$ and let $A_{2}$ be the kernel of the map $x \longmapsto 2 x$ of $A$. Then he showed, using his work $[\mathbf{M a 9}$ ], that there are more irreducible $\sigma$-representations and constructed a natural imbedding of $G / A^{2} \times A_{2}$ into the $\sigma$-dual of $G$, and further that this imbedding is a bijection if and only if $A^{2}$ is closed in $A$. Let $G_{2}$ be the subgroup of elements of order 2 in $G$. Then he showed that, in the case when $A^{2}$ is closed in $A$, for each character $\chi$ of $G_{2}$ there is a unique irreducible $\sigma$-representation $W^{\chi}$ of $G$ that coincides on $G_{2}$ with a multiple of $\chi$, and the $W^{\chi}$ are all the irreducible $\sigma$-representations of $G$; if $W_{0}$ is the one for $\chi=\chi_{0}(a, \xi)=\langle a, \xi\rangle$, and $\operatorname{Sp}_{0}(G)$ is the subgroup of the symplectic group fixing $\chi_{0}$, then we have a natural projective 
representation of $\operatorname{Sp}_{0}(G)$ in the space of $W_{0}^{\chi_{0}}$. In this manner he obtained the complete result involving the canonical projective representation of the symplectic group of $G$ without any restrictions on $A$ except that $A^{2}$ be closed in $A$.

It is possible to describe Mackey's uniqueness of the Schrödinger representation in a more general framework $[\mathbf{D V}][\mathbf{M u}]$. Let $G$ be any separable locally compact abelian group. If $m$ is a multiplier for $G$, then

$$
m^{\sim}(x, y)=\frac{m(x, y)}{m(y, x)}
$$

can be proved to be a continuous bicharacter (a character in each argument); we shall say that $m$ is a Heisenberg multiplier if $m^{\sim}$ is symplectic, i.e., the map from $G$ to $\widehat{G}$ induced by $m^{\sim}$ is an isomorphism. If $G$ is 2-regular, there is a unique symplectic multiplier in the cohomology class of $m$ so that $m$ itself may be assumed to be symplectic. The most natural and general form of Mackey's uniqueness theorem without any assumption on $G$ is now the following:

TheOREM (MACKeY). If $m$ is a Heisenberg multiplier for the separable locally compact abelian group $G$, then $G$ has a unique irreducible $m$-representation, and every m-representation of $G$ is a direct sum of copies of this unique irreducible one.

This theorem of Mackey has been used by Mumford $[\mathbf{M u}]$ in his Tata lectures on theta functions quite systematically. The passage from $m$ to $m^{\sim}$ is quite natural; in fact, if $H_{m}$ is the extension of $G$ by the circle group $T$ so that

$$
1 \longrightarrow T \longrightarrow H_{m} \longrightarrow G \longrightarrow 1
$$

is exact, the commutator map $x, y \longmapsto x y x^{-1} y^{-1}$ descends to a map $G \times G \longrightarrow T$ which works out to $m^{\sim}$. The Heisenberg condition on $m$ is that $m^{\sim}$ gives rise to a perfect pairing.

Two additional remarks are appropriate here. First, the work of Shale, although restricted to the real case, is more general because the groups are no longer locally compact; indeed, the situation is inspired by quantum field theory, as I have discussed earlier. Here the commutation rules do not have a unique representation and so it is not immediately clear how we get the projective representation of the symplectic group; indeed, if we start with an invariant measure class associated to a real infinite dimensional Hilbert space (one has to be careful here as the measures are not defined on the real Hilbert space), a symplectic automorphism will change the measure class into another one which is often singular with respect to it. One has to introduce a restricted symplectic group whose elements preserve the measure class. For some good choices of the measure class Shale proved that the corresponding restricted symplectic group is the subgroup of the full symplectic group of maps $s$ such that $\left(s^{*} s\right)^{1 / 2}$ is of the form $I+S$ where $S$ is of the Hilbert-Schmidt class. Shale's theory was later extended to the $p$-adic case by $[\mathbf{Z}]$. Much remains to be done here.

The second remark concerns with what Weil did. He did not stop with the projective representation of the symplectic group; in the local and adleic cases that he treated, he proved that the projective representation in question becomes an ordinary representation when we pass to the 2 -fold cover of the symplectic group, the so-called metaplectic group; Shale had done this for $\mathbf{R}$. The lifted unitary representation is often called the metaplectic representation. I do not know if Mackey had any results on the order of the multiplier of his representation of the 
symplectic group in the case of a general locally compact separable abelian A. This seems to be an interesting question in the general framework that Mackey initiated.

\section{Foundations of Physics: hidden variables and the Mackey-Gleason theorem}

Mackey's approach to science in general, and physics in particular, was one of great curiosity combined with great restraint. Unlike many mathematicians he was not trying to solve all the problems of the physicists, nor was he trying to tell the physicists what they were or should be doing. He was very much more modest, content to understand and interpret the physicists' conception of the world from a mathematician's point of view.

Hidden variables, the Mackey problem of measures on questions, and Gleason's theorem. With the development of quantum theory in the 1920's it became clear that an entirely novel view of physical reality had emerged as the foundation of the new theory, a view that contrasted very sharply with the causal and deterministic view of spacetime events and their description that had been the basis hitherto for all classical physics. In the quantum theoretic view, in even the most idealized state of a quantum system, one can in principle determine only the statistical distributions of the various physical quantities, because of the inherently uncontrollable interaction between the phenomena that are being measured and the measuring apparatus. Although it is often possible to arrange matters (called preparation of state) so that a particular observable has a sharply defined value (has no dispersion) or at least has arbitrarily small dispersion, this can be done only at the cost of other observables having large dispersions. There is no state in which all observables have sharply defined values or arbitrarily small dispersions. Obviously this circumstance created a huge amount of discussion of fundamental matters, among which discussions in the various Solvay conferences between Einstein and Bohr figured very prominently [Bo]. The basic question became whether the quantum mechanical description of reality was complete. When von Neumann wrote his great book in 1933 [vN2] he gave a systematic mathematical and physical discussion of this question at the most general level.

Like every one who came after von Neumann, Mackey was profoundly influenced by von Neumann's great 1932 classic [vN2]. The question whether the quantum mechanical description of reality is complete can be made accessible to a mathematical treatment by posing it as the problem of hidden variables in quantum mechanics: is it possible that the statistical features of the usual model for quantum theory are due to our incomplete knowledge of the state of the system, and arise because of averaging over a space of parameters (the hidden variables)? In quantum mechanics the states are unit vectors up to a phase in a Hilbert space, and it is a remarkable feature of the theory that no matter what the state is, there are observables which do not have a sharply defined value, or, have positive dispersion. In other words the states of quantum mechanics are not dispersion-free. If now there are hidden parameters, the states defined by specific values of the hidden parameters would define dispersion-free states (although these would be highly idealized and may not be accessible to any practical technology); the situation would be very much like in statistical mechanics. The quantum states would then be mixtures of these idealized dispersion-free states, and so we would be able to write the quantum states as convex linear combinations of other states. Therefore, to 
put to rest the possibility of an explanation by hidden parameters of the statistics derivable from the standard model of quantum mechanics, one must answer in the affirmative the following mathematical question: using the most general definition of a state of a quantum system, is it true that the quantum states defined by the unit vectors cannot be written as convex combinations of other states, i.e., they are the extreme points of the convex set of all states? The answer to this problem of course depends on the general definition of a state of a quantum system, and clearly one has to base the argumentation on the widest interpretation of the notion of the quantum state in order to have the most convincing result. The answer, as von Neumann saw it, was a resounding negative [vN2], and after many variants and modifications von Neumann's analysis still remains the best treatment of this question (see the wonderful reprint collection $[\mathbf{W Z}]$ where the various articles on this question are reprinted). The approach of von Neumann was to observe that no matter what models are used - the usual quantum model, the hidden parameters model, or any other - for theoretical purposes all one needs to consider are the expectation values of the observables measured in the various states of the system. Hence one can identify the state of the system with the expectation value functional on the set of bounded observables in the system. Now, in the usual model, whose statistics form the object of scrutiny, the set of bounded observables is $B_{\mathbf{R}}(\mathcal{H})$, the set of Hermitian elements of the algebra $B(\mathcal{H})$ of all bounded operators. $B_{\mathbf{R}}(\mathcal{H})$ is not an algebra but is a real vector space and contains the square of each element. The properties that von Neumann assumed for the expectation functional

$$
E: A \longmapsto E(A) \quad\left(A \in B_{\mathbf{R}}(\mathcal{H})\right)
$$

were as follows:

(a) $E$ is linear

(b) $E(1)=1$

(c) $E\left(A^{2}\right) \geq 0$ for all $A$

(d) $E$ is continuous in the strong operator topology.

He then determined all such functionals, and showed that the pure states, defined as the extreme points of the convex set of states, are precisely those given by the usual unit vectors up to a phase of the Hilbert space. Let us define a density matrix as a bounded positive self adjoint operator of trace class and having trace 1 . Then von Neumann's analysis showed that the expectation functionals are precisely those of the form

$$
E^{U}: A \longmapsto \operatorname{Tr}(A U)=\operatorname{Tr}\left(U^{1 / 2} A U^{1 / 2}\right) .
$$

The correspondence

$$
U \longmapsto E^{U}
$$

is convex and one-one, and so, as the density matrices form a convex set, we have only to determine the extreme points of this set. It is not difficult to see that the extreme points are the one dimensional projections and so are labeled by unit vectors up to a phase, thus answering the question of hidden parameters in the negative.

If one wants to make a critical assessment of von Neumann's analysis, the place to begin with is the axiomatization of the expectation functional. Now, if $A$ and $B$ are quantum observables, von Neumann used the ensemble method characteristic of thermodynamics to give an operational definition of $A+B$ even when $A$ and $B$ are not simultaneously observable. This allowed him to assume that the expectation 
value is additive: $E(A+B)=E(A)+E(B)$. The assumption of countable additivity for $E$ is a consequence of the strong continuity and may be regarded as a regularity assumption; we shall temporarily set this aside and concentrate just on the linearity. If $A$ and $B$ are simultaneously measurable, then there is no difficulty in measuring $A+B$ and obtaining the additivity of the expectation value, since $A$ and $B$ are random variables on a single classical probability space. When $A$ and $B$ are not simultaneously observable, the definition of $A+B$ as well as the additivity of the expectation value are less convincing. Indeed, in many instances, the distributions of $A, B$, and $A+B$ are such that they cannot be derived from a single probability distribution on the plane $\mathbf{R}^{2}$. Moreover, this is not some arcane issue as von Neumann himself observed: the very definition of the Hamiltonian in the form

$$
H=\frac{1}{2}\left(p_{1}^{2}+p_{2}^{2}+\cdots+p_{n}^{2}\right)+V\left(q_{1}, q_{2}, \ldots, q_{n}\right)
$$

shows that we are concerned precisely with this type of situation even in the simplest applications of quantum theory. The $p_{i}$ and $q_{j}$ commute among themselves and so

$$
A=\frac{1}{2}\left(p_{1}^{2}+p_{2}^{2}+\cdots+p_{n}^{2}\right), \quad B=V\left(q_{1}, q_{2}, \ldots, q_{n}\right)
$$

are individually well defined, but $H=A+B$ requires a stretch of imagination. Even in the case of the usual model, $A$ and $B$ are defined very simply as self adjoint operators, but $A+B$ is only symmetric on the intersection of the domains of $A$ and $B$; that it has a unique self adjoint extension is true under very general circumstances, as was first proved by Kato, but this is not an obvious result.

The starting point of Mackey's analysis is that one should assume additivity of the expectation only for commuting observables. To discuss the structure of the expectation functionals we may restrict ourselves to what Mackey called questions, namely, observables which take only the two values 0,1 . These are represented by projections in the Hilbert space. The expectation value thus defines a function

$$
\mu: P \longmapsto \mu(P)
$$

on the set of projections and having values in the interval $[0,1]$ of the real line. If $P$ and $Q$ are orthogonal projections, then $P+Q$ is again a projection and it is clear that we have

$$
\mu(P+Q)=\mu(P)+\mu(Q)
$$

so that $\mu$ is finitely additive. In other words $\mu$ is a finitely additive probability measure on projections. If $P$ and $Q$ are projections which are not orthogonal, $P+Q$ may not be a projection; it is still a bounded observable and so has an expectation value, but we do not assume that the expectation of $P+Q$ is the sum $\mu(P)+\mu(Q)$ as part of the data defining the state. For any bounded Hermitian $A$ with spectral measure $P^{A}$,

$$
\mu^{A}: S \longmapsto \mu\left(P_{S}^{A}\right) \quad(S \text { a Borel set } \subset \mathbf{R})
$$

is a finitely additive probability measure with bounded support on the real line and we can define the expectation value of $A$ as

$$
E^{\mu}(A)=\int_{\mathbf{R}} t d \mu^{A}(t) .
$$

(There is no difficulty in defining integrals of bounded functions with respect to finitely additive measures). It is then easy to see that $E^{\mu}$ satisfies the properties of 
von Neumann except that the additivity is asserted only for commuting operators:

$$
E^{\mu}(A+B)=E^{\mu}(A)+E^{\mu}(B) \quad(A B=B A) .
$$

Conversely, any functional $E$ on $B_{\mathbf{R}}(\mathcal{H})$ with the properties assumed by von Neumann except that additivity is assumed only for commuting elements, arises from a unique $\mu$.

We shall call countably additive probability measures on the lattice of projections Mackey states. The original states in von Neumann's analysis will be called von Neumann states. The finitely additive probability measures on the lattice of projections are called generalized Mackey states. If $\mu$ is a generalized Mackey state, then for any self adjoint operator $A$ with spectral measure $P^{A}$,

$$
E^{\mu}(A)=\int_{\mathbf{R}} t d \mu^{A}(t) .
$$

It is far from obvious that $E^{\mu}$ thus defined is unrestrictedly linear for a Mackey state $\mu$, generalized or otherwise, nor is it clear why it should be strongly continuous. In other words a Mackey state need not be (define) a von Neumann state.

Mackey states which are von Neumann states exist in abundance. If $U$ is a density matrix

$$
\mu^{U}: P \longmapsto \operatorname{Tr}(P U)=\operatorname{Tr}\left(U^{1 / 2} P U^{1 / 2}\right)
$$

is a Mackey state which is even a von Neumann state because of the linearity and continuity properties of Tr. We thus have three levels of states of increasing generality:

A. von Neumann states $E^{U}$, defined by density matrices $U$

B. countably additive Mackey states, defined by countably] additive probability measures on the lattice of projections of the Hilbert space $\mathcal{H}$

C. the generalized Mackey states, defined by finitely additive probability measures on the lattice of projections of $\mathcal{H}$.

When he arrived at this point in his analysis, Mackey asked his colleague Gleason if it could be proved that any countably additive probability measure on the lattice of projections is of the form $\mu^{U}$ for a density matrix $U$. As he told me in Seattle, Gleason almost immediately reduced this question to the case when the underlying Hilbert space is real and of dimension 3 (the result requires the dimension to be $\geq 3$, there are counter examples in dimension 2); very soon afterwards he proved the result in dimension $3[\mathbf{G l e}]$. Thus, in dimension $\geq 3$, Mackey states are von Neumann states.

Let us now use Gleason's result and complete the proof of a sharpening of Mackey's analysis, namely that even if we take the state to be the most general one of level $C$, there are none which are dispersion-free. It is first of all easy to see that if a finitely additive $\mu$ is dispersion-free then it has to be two-valued, i.e., it takes only the two values 0 and 1 ; indeed, if for some projection $P$ we have $0<\mu(P)=p<1$, then the observable $P$ takes just the two values 0 and 1 with respective probabilities $p$ and $1-p=q$; it is thus a classical binomial variable and its dispersion is $p q>0$. The question therefore becomes the following: are there any two-valued finitely additive probability measures on the lattice

$\mathcal{L}(\mathcal{H})$ of projections on a Hilbert space? We shall now show that Gleason's theorem implies that if $\operatorname{dim}(\mathcal{H}) \geq 3$ then there are no two-valued finitely additive probability measures on $\mathcal{L}(\mathcal{H})$ and hence no dispersion-free states. In fact, if $3 \leq \operatorname{dim}(\mathcal{H})<\infty$, then all finitely additive measures on $\mathcal{L}(\mathcal{H})$ are automatically 
countably additive, and so Gleason's theorem shows that $\mu=\mu^{U}$ for a density matrix $U$; if $\phi$ is a unit vector and $P_{\phi}$ is the projection on the one dimensional space spanned by $\phi$, then $\mu^{U}\left(P_{\phi}\right)=(U \phi, \phi)$, and this, as a function of $\phi$ is continuous and takes all values between 0 and 1 , thus certainly not two-valued. If $\operatorname{dim}(\mathcal{H})=\infty$, the isomorphism $\mathcal{H} \simeq \mathcal{H} \otimes \mathbf{C}^{3}$ shows that we can imbed $\mathcal{L}\left(\mathbf{C}^{3}\right)$ inside $\left.\mathcal{L}^{(} \mathcal{H}\right)$, and so, if $\mu$ is two-valued on $\mathcal{L}(\mathcal{H})$, the restriction of $\mu$ to the image of $\mathcal{L}\left(\mathbf{C}^{3}\right)$ under the above imbedding is a two-valued measure on $\mathcal{L}\left(\mathbf{C}^{3}\right)$ the existence of which we have just shown to be impossible. Thus the non existence of two valued finitely additive measures on $\mathcal{L}(\mathcal{H})$ is proved also when $\operatorname{dim}(\mathcal{H})=\infty$. Actually this argument yields the much more general result that if the quantum logic contains $\mathcal{L}\left(\mathbf{C}^{n}\right)$ as a sublogic for some $n \geq 3$, it does not admit any two valued measures, and hence no dispersion free states.

The reader should note the contrast with Boolean algebras where there are always two-valued measures. In the Boolean algebra case this result, going back to Stone, is at the very foundation of set theory and Boolean logic, indeed, it is equivalent to the axiom of choice when properly formulated; it is therefore remarkable that the Mackey-Gleason analysis led to the discovery that the logic of quantum mechanics, with its characteristic feature of the complementarity principle, is at a profound variance with classical logic.

Unfortunately, by the time these results were obtained, von Neumann was dead, but one can be sure however that they would have pleased him. There is also another point, namely, the question of the possible extension of Gleason's theorem to the lattice of projections in other von Neumann algebras. The Gleason theorem has indeed been extended to the case of the lattice of projections on fairly arbitrary von Neumann algebras with some mild restriction. The subject is a part of non commutative integration which was pioneered by von Neumann and which has eventually led to the modern theory of non commutative geometry.

One final remark may be made. In recent years there has been a flurry of activity and results, both experimental and theoretical, regarding the hidden variables question. The theoretical results (Bell's inequality etc) do not change anything in the von Neumann-Mackey-Gleason treatment; they are less general, being dependent on some model or the other. Their significance lies in the fact that they brought the question of consistency and completeness of quantum mechanics to the realm of the experimenter, and led to some beautiful experiments $[\mathbf{A G}]$. For all these matters see $[\mathbf{B L}][\mathbf{V a 2}][\mathbf{W Z}]$.

Quantum field theory. When physicists first started to develop quantum field theory in the early 1930's after Dirac's theory of radiation (see [vN2]), the idea was to apply the procedure of quantization to a classical system of infinitely many degrees of freedom, such as the Maxwell electromagnetic field. The classical description then involved an infinite dimensional phase space and so one had an infinite number of canonical variables $q_{1}, q_{2}, \ldots, p_{1}, p_{2}, \ldots$. But after some time people realized that the commutation rules no longer have a unique representation and so the procedure of quantization and dynamical evolution became ambiguous. In the early 1950's Friedrichs, and somewhat later, Segal, discussed why the commutation rules have different representations. In his Seattle lectures Mackey put this problem in a framework closely related to his 1949 paper [Ma1] in a formulation that made it crystal clear why these new phenomen were coming up. He had 
two abelian standard Borel groups $G$ and $H$ in a Borel duality

$$
\langle\cdot, \cdot\rangle: G \times H \longrightarrow S^{1}
$$

and framed the basic question as the construction of pairs $(U, V)$ where $U, V$ are unitary representations of $G, H$ respectively, satisfying the Weyl relation:

$$
U(g) V(h)=\chi(g, h) V(h) U(g) \quad((g, h) \in G \times H) .
$$

The difference from the classical situation is that the groups are $G, H$ are no longer locally compact, and in fact are usually defined by some topological data that makes them infinite dimensional, reflecting the fact that the classical system has infinitely any degrees of freedom. If $G$ has an invariant measure class under translations, then one can construct an associated Schrödinger representation; but now it is no longer reasonable to expect that the invariant measure class is unique, and so one gets different Schrödinger representations corresponding to the different invariant measure classes.

I do not know if he pursued his way of formulating quantum field theory beyond these preliminaries, especially as an alternative to the usual treatments of what is now called constructive quantum field theory. It would be interesting to see if a general approach along the lines of his ideas would throw additional light on the problems of quantum field theory. His book [Ma4] contains a systematic exposition of an extensive part of quantum theory from the point of view of unitary group representations. In his later years he gave some detailed expositions of his approach to the group theoretic foundations of quantum theory that touched on a variety of topics, including gauge theory. The interested reader should look up some of these [Ma13]; the article (a) in this set of references was the one that I came across when I was a graduate student and it changed my entire perspective instantly.

Canonical commutation relation and quantum stochastic calculus. At the risk of some digression I shall point out that there are connections between the field commutation rules and what is now called quantum stochastic calculus. The general philosophy is that the dynamics of any irreversible quantum system is mediated by a one-parameter semigroup $\left\{T_{t}, t \geq 0\right\}$ of linear, unital and completely positive maps on a $C^{*}$ algebra $\mathcal{A}$ with identity with the infinitesimal generator $\mathcal{L}$. For brevity and simplicity we confine ourselves here to a finite level system where $\mathcal{A}=\mathcal{B}(\mathcal{H})$ is the algebra of all operators on a finite dimensional Hilbert space $\mathcal{H}$ and the map $t \mapsto T_{t}$ is continuous. It is known that $\mathcal{L}=\mathcal{L}_{0}+\mathcal{L}_{1}$ where $\mathcal{L}_{0}$ describes quantum fluctuations from a reversible Heisenberg or Schrödinger dynamics whereas $\mathcal{L}_{1}$ describes the dissipation part which contributes to irreversibility. It is a part of the general theory that the semigroup $\left\{T_{t}\right\}$ is a coarse-grained version of the unitary evolution $\left\{U_{t}\right\}$ of a larger quantum system which is made of the original system and a bath (or noise) consisting of a collection of harmonic oscillators. The Hilbert space of such a bath is usually described by a boson Fock space (or a second quantization space). Quantum stochastic calculus $[\mathbf{H P}][\mathbf{M e}][\mathbf{P 2}][\mathbf{S G}]$ provides a framework which facilitates the task of making this description mathematically precise.

To any Hilbert space $\mathcal{K}$ associate the boson Fock space $\Gamma(\mathcal{K})$ defined by

$$
\Gamma(\mathcal{K})=\mathbf{C} \oplus \mathcal{K} \oplus \mathcal{K}^{2} \oplus \cdots \oplus \mathcal{K}^{n} \oplus \cdots
$$

where $\mathcal{K}^{n}$ is the $n$-fold symmetric tensor product of copies of $\mathcal{K}$. We have the usual annihilation operators and their adjoints called creation operators whose domains 
include a common core $\mathcal{D}$ and satisfy the canonical commutation rules. Then the Hilbert space $\Gamma(\mathcal{K})$ is an example of a continuous tensor product admitting the factorization

$$
\Gamma(\mathcal{K}) \cong \Gamma\left(L^{2}[0, t] \otimes \mathbf{K}\right) \otimes \Gamma\left(L^{2}[t, \infty] \otimes \mathbf{K}\right) \quad \forall t \geq 0 .
$$

We introduce the 'system + bath' Hilbert space

$$
\widetilde{\mathcal{H}}=\mathcal{H} \otimes \Gamma(\mathcal{H})=\mathcal{H}_{t]} \otimes \mathcal{H}_{[t} \quad \forall t \geq 0 .
$$

where $\mathcal{H}_{t]}=\mathcal{H} \otimes \Gamma\left(L^{2}[0, t] \otimes \mathbf{K}\right), \mathcal{H}_{[t}=\Gamma\left(L^{2}[t, \infty] \otimes \mathbf{K}\right) \forall t \geq 0$ and $\mathcal{H}_{0]}=\mathcal{H}$. A family $\{X(t), t \geq 0\}$ in $\widetilde{\mathcal{H}}$ is said to be an adapted process if $X(t)=X_{0}(t) \otimes I_{[t} \forall t$ where $X_{0}(t)$ is an operator in $\mathcal{H}_{t]}$ and $I_{[t}$ is the identity operator in $\mathcal{H}_{[t}$. Heuristically, such operators $X(t)$ describe observables that depend on the evolution of the system and the bath up to time $t$. Relative to a suitable family of conditional expectations the creation and annihilation processes (defined by following the intuition of free field theory) exhibit martingale-like properties and hence permit a definition of quantum stochastic integrals along the lines of the classical theory of stochastic integration in the sense of Ito. For a full account of this quantum Ito's formula we refer to $[\mathbf{H P}][\mathbf{P 2}]$. The classical Ito's formula is a consequence of this quantum version.

We now look for a unitary operator-valued adapted process $U=\left\{U_{t}, t \geq 0\right\}$ which is a solution of the quantum stochastic differential equation of the exponential type. It is a theorem that there exists a unique operator-valued adapted process satisfying this equation and the semigroup $\left\{T_{t}\right\}$ with generator $\mathcal{L}$ is obtained from

$$
T_{t}(X)=\mathbf{E}_{0]} U_{t}\left(X \otimes I_{[0}\right) U_{t}^{\dagger} \quad \forall t \geq 0, \quad X \in \mathcal{B}(\mathcal{H}) .
$$

In other words $T_{t}$ is the vacuum conditional expectation of the Heisenberg evolution by the unitary operator $U_{t}$ in the system plus noise Hilbert space $\widetilde{\mathcal{H}}$. For more detailed accounts and current developments we refer to $[\mathbf{M e}][\mathbf{P 2}][\mathbf{S G}]$.

Concluding remarks. I have restricted myself only to those parts of Mackey's work with which I have some familiarity. I have not discussed his work and ideas in many other areas such as ergodic theory which have been both profound and influential. In fact, his notion of virtual subgroups was the first hint that there was a deep theory hidden behind ergodic phenomena. It must be clear to the reader even from my rather selective discussion of his work that Mackey was a thinker of exceptional depth and originality, and understood at a very fundamental level the relation between the theory of representations of groups and homogeneous spaces and modern physics. Nowadays many people are searching for a true generalization of the geometry of the physical world that would accommodate both gravity and quantum theory-which will be non commutative at the Planck scale and be a very good approximation to the Riemann-Einstein geometry of spacetime at the ordinary scale. Although he himself did not seriously participate in these developments I believe that Mackey's ideas and themes will find a resonance in this new frontier. It is the task of the younger generation to discover this and move forward.

\section{Mackey References}

[Ma1] G. W. Mackey, Infinite-dimensional group representations, Bull. Amer. Math. Soc., 69 (1963), 628-686. 
[Ma2] - The theory of unitary group representations. Based on notes by James M. G. Fell and David B. Lowdenslager of lectures given at the University of Chicago, Chicago, Ill., 1955. Chicago Lectures in Mathematics. University of Chicago Press, Chicago, Ill.London, 1976.

[Ma3] $\longrightarrow,(a) \ldots$ Induced representations of groups and quantum mechanics, W. A. Benjamin, Inc., New York-Amsterdam; Editore Boringhieri, Turin, 1968.

(b) - Mathematical foundations of quantum mechanics. With a foreword by A. S. Wightman. Reprint of the 1963 original. Dover Publications, Inc., Mineola, NY, 2004.

[Ma4] - (a) Unitary group representations in physics, probability, and number theory, Mathematics Lecture Note Series, 55. Benjamin/Cummings Publishing Co., Inc., Reading, Mass., 1978. Second edition. Advanced Book Classics. Addison-Wesley Publishing Company, Advanced Book Program, Redwood City, CA, 1989.

(b) - Harmonic analysis as the exploitation of symmetry-a historical survey, Bull. Amer. Math. Soc. (N.S.) 3 (1980), no. 1, part 1, 543-698.

(c) _ Harmonic analysis and unitary group representations: the development from 1927 to 1950. L'mergence de l'analyse harmonique abstraite (1930-1950), (Paris, 1991), 13-42, Cahiers Sm. Hist. Math. Sr. 2, 2, Univ. Paris VI, Paris, 1992.

$(d)$, The scope and history of commutative and noncommutative harmonic analysis, History of Mathematics, 5. Amer. Math. Soc., Providence, RI; London Mathematical Society, London, 1992.

[Ma5] _ A theorem of Stone and von Neumann, Duke Math. J. 16 (1949), 313-326.

[Ma6] Imprimitivity for representations of locally compact groups. I, Proc. Nat. Acad. Sci. U. S. A. 35 (1949), 537-545.

[Ma7] _ Induced representations of locally compact groups. I, Ann. of Math. (2) 55 (1952), 101-139.

[Ma8] - Induced representations of locally compact groups. II. The Frobenius reciprocity theorem, Ann. of Math. (2) 58 (1953), 193-221.

[Ma9] - Unitary representations of group extensions. I, Acta Math. 99 1958, 265-311.

[Ma10] - On induced representations of groups, Amer. J. Math. 73 (1951), 576-592.

[Ma11] L Les ensembles borliens et les extensions des groupes, J. Math. Pures Appl. (9) 36 (1957), 171-178.

[Ma12] Some remarks on symplectic automorphisms, Proc. Amer. Math. Soc. 16, 1965 393-397.

[Ma13] (a) , Quantum mechanics and Hilbert space, Amer. Math. Monthly 64 (1957) no. 8, part II, $45-57$.

(b) - Quantum mechanics from the point of view of the theory of group representations, Applications of group theory in physics and mathematical physics (Chicago, 1982), 219-253, Lectures in Appl. Math., 21, Amer. Math. Soc., Providence, RI, 1985. (c) - Weyl's program and modern physics, in Differential Geometrical Methods in Theoretical Physics (Como, 1987), 11-36, NATO Adv. Sci. Inst. Ser. C Math. Phys. Sci., 250, Kluwer Acad. Publ., Dordrecht, 1988.

(d) , Axiomatics of particle interactions, Proceedings of the International Quantum Structures Association, Part I (Castiglioncello, 1992). Internat. J. Theoret. Phys. 32 (1993), no. 10, 1643-1659.

(e) — The relationship between classical mechanics and quantum mechanics, Perspectives on quantization (South Hadley, MA, 1996), 91-109, Contemp. Math., 214, Amer. Math. Soc., Providence, RI, 1998.

\section{Other References}

[AG] A. Aspect and P. Grangier, Experimental tests of Bell's inequalities, in Advances in Quantum Phenomena, (eds.) E. G. Beltrametti and J-M. L. Lévy-Leblond., NATO ASI Ser. B Vol 347 (1995), 201-213.

[BL] E. G. Beltrametti and J-M. L. Lévy-Leblond (eds.), Advances in Quantum Phenomena, NATO ASI Ser. B Vol 347 (1995).

[Ba] V. Bargmann, On unitary ray representations of continuous groups, Ann. of Math., 59 (1954), 1-46. 
[Bo] N. Bohr, Discussion with Einstein on epistemological problems in atomic physics, in Albert Einstein : Philosopher-Scientist, (ed.) P. A. Schilpp, 200-241, The Library of Living Philosophers, Evanston (1949); reprinted in [WZ] below (pp. 9-49).

[Bos1] S. K. Bose, The Galilean group in 2+1 space-times and its central extensions, Comm. Math. Phys. 169 (1995), 385-395.

[Bos2] S. K. Bose, Representations of the(2+1)-dimensional Galilean group, J. Math. Phys. 36 (1995), 875-890.

[Br1] F. Bruhat, Sur les representations induites des groupes de Lie, Bull. Soc. Math. France, 84 (1956), 97-205.

[Br2] F. Bruhat, Distributions sur un groupe localement compact et applications à l'étude des representations des groupes p-adiques, Bull. Math. Soc. France, 89 (1961), 43-75.

[CDLL] G. Cassinelli, E. De Vito, P. Lahti and A. Levrero, Symmetry of the Quantum State Space and Group Representations, Rev. Math. Phys 10 (1998), 893-924.

[CHC1] Charles H. Conley, Little group method for smooth representations of finite length, Duke Math. J. 79 (1995), no. 3, 619-666.

[CHC2] Charles H. Conley, Geometric realizations of representations of finite length, Rev. Math. Phys. 9 (1997), no. 7, 821-851. II. Pacific J. Math. 183 (1998), no. 2, 201-211.

[CTV] G. Cassinelli, P. Truini and V. S. Varadarajan, Hilbert space representations of the Poincar group for the Landau gauge, J. Math. Phys. 32 (1991), no. 4, 1076-1090.

[CCTV1] C. Carmeli, G. Cassinelli, A. Toigo and V. S. Varadarajan, Unitary representations of super Lie groups and applications to the classification and multiplet structure of super particles, Comm. Math. Phys., 263 (2006), 217-258.

[CCTV2] C. Carmeli, G. Cassinelli, A. Toigo and V. S. Varadarajan, Induced representations of super Lie groups, In preparation.

[DV] T. Digernes and V. S. Varadarajan, Models for the irreducible representation of a Heisenberg group, Infinite dimensional analysis, quantum probability, and related topics, 7 (2004), 527-546.

[De] E. De Vito, Thesis, University of Genoa.

[Di] P. T. Divakaran, Symmetries and quantization: structure of the state space, Rev. Math. Phys.9 (1994), 167-205.

[du C] F. du Cloux, Extensions entre représentations unitaires irreductibles des groupes de Lie nilpotents, Asterisque, 124-125 (1985), 129-211.

[E] E. G. Effros, Transformation groups and $C^{*}$-algebras, Ann. of Math. (2) 81 1965, 38-55.

[Gle] A. M. Gleason, Measures on the closed subspaces of a Hilbert space, Journal of Mathematics and Mechanics, 6 (1957), 885-894.

[Gl] J. Glimm, Locally compact transformation groups, Trans. Amer. Math. Soc., 101 (1961), 124-138; Type I C*-algebras, Ann. of Math., 73 (1961), 572-612.

[Gui1] A. Guichardet, Extensions des représentations induites des produits semidirects, J.für reine angew. Math, 310 (1979), 7-32.

[Gui2] A. Guichardet, Représentations de longeur finie des groupes de Lie inhomogènes, Astérisque, 124-125 (1985), 212-252.

[Hal] P. R. Halmos, Introduction to Hilbert Space and the theory of Spectral Multiplicity, Chelsea, 1957.

[Ha1] Harish-Chandra, On the irreducibility of induced representations, Unpublished manuscript, c. 1970.

[Ha2] Harish-Chandra, Eisenstein series over finite fields, Proceedings of a conference in honor of Professor Marshall Stone held at the University of Chicago, May 1968, (Ed.) F. E. Browder, pp. 76-88, Springer-Verlag, 1970.

[HP] R. L. Hudson and K. R. Parthasarathy, Quantum Ito's formula and stochastic evolutions, Commun. Math. Phys. 93, 301-23 (1984).

[KV] Johan A. C. Kolk and V. S. Varadarajan, On the transverse symbol of vectorial distributions and some applications to harmonic analysis, Indag. mathem., N. S., 7 (1996), 67-96.

[Me] P. A. Meyer, Quantum Probability for Probabilists, Lecture Notes in Mathematics 1538, Springer Verlag, Heidelberg (1993).

[Moo1] C. C. Moore, Group extensions of p-adic and adelic linear groups, Inst. Hautes tudes Sci. Publ. Math. No. 351968 157-222. 
[Moo2] C. C. Moore, Trans. Amer. Math. Soc., 113 (1964), 64-86.

$[\mathrm{Mu}]$ D. Mumford, Tata Lectures on Theta. III, Progress in Mathematics, 97, Birkhäuser, 1991 (with the collaboration of M. Nori and P. Norman).

[N] N. Nakanishi, Prog. Theor. Phys., 49. (1973), 640; Suppl., 51, 1.

[vN1] J. von Neumann, Die Eindeutigkeit der Schrödingerschen Operatoren, Math. Ann., 104 (1931), 570-578.

[vN2] J. von Neumann, Mathematische Grundlagen der Quantenmechanik, Springer-Verlag, Berlin, 1932. The English translation is Mathematical Foundations of Quantum Mechanics, Robert T. Beyer (Translator) Princeton University press, 1955.

[NC] M. A. Nielsen and I. L. Chuang, Quantum Computation and Quantum Information, Cambridge University Press (2000).

[PRV] K. R. Parthasarathy, R. Ranga Rao and V. S. Varadarajan, Representations of complex semi-simple Lie groups and Lie algebras, Ann. of Math. (2) 85-(1967), 383-429.

[P1] K. R. Parthasarathy, Quantum Computation, Quantum Error Correctng Codes and Information Theory, TIFR Lecture Notes by Amitava Bhattacharya, Narosa Publishing House, New Delhi (2006).

[P2] K. R. Parthasarathy, An Introduction to Quantum Stochastic Calculus, Birkhauser Verlag, Basel (1992).

[R] G. Rideau, J. Math. Phy., 19, (1978), 1627.

D. Shale, Linear symmetries of free boson fields, Trans. Amer. Math. Soc. P103 (1962), 149-167.

[SG] K. B. Sinha and D. Goswami, Quantum Stochastic Processes and Noncommutative Geometry, Cambridge University Press, (2007).

[St] M. H. Stone, Linear transformations in Hilbert Space. III. Operational methods and group theory, Proc. Nat. Acad. Sci. USA, 16 (1930), 172-175.

[Va1] V. S. Varadarajan, Supersymmetry for mathematicians: an introduction, Courant Lecture Notes in Mathematics, 11. New York University, Courant Institute of Mathematical Sciences, New York; Amer. Math. Soc., Providence, RI, 2004.

[Va2] V. S. Varadarajan, Geometry of Quantum Theory, Second edition. Springer-Verlag, New York, 1985.

[Va3] V.S. Varadarajan, Probability in physics and a theorem on simultaneous observability, Comm. Pure and Appl. Math., 15 (1962), 189-217.

[vW] B. L. van der Waerden, Sources of Quantum Mechanics, Dover, 1967.

[We1] A. Weil, Sur certaines groupes d'opérateurs unitaires, Acta Mathematica, 111 (1964), 143-211.

[We2] A. Weil, Sur la formule de Siegel dans la théorie des groupes classiques, Acta Mathematica, 113 (1965), 1-87.

[W] H. Weyl, Group Theory and Quantum Mechanics, Dover, 1931.

[WZ] J. A. Wheeler and W. H. Zurek, Quantum Theory and Measurement, Princeton University Press, Princeton, 1983.

[Wi] E. P. Wigner, Unitary representations of the inhomogeneous Lorentz group, Ann. of Math., 40 (1939), 149-204.

[Z] E. P. Wigner, Representations of commutations relations for p-adic systems of infinitely many degrees of freedom. Print-91-0246. Steklov Math. Inst. Moscow.

University of California, Los Angeles, USA

E-mail address: vsv@math.ucla.edu 